\title{
VALIDATION OF THE FEMAXI/SIMMER COUPLING BY USING THE E9 CABRI-2 EXPERIMENT
}

\author{
Simone Gianfelici ${ }^{1}$, Sara Perez-Martin ${ }^{1}$, Werner Pfrang${ }^{1}$, Thomas Schulenberg ${ }^{1}$, Andrei \\ Rineiski $^{1}$ \\ ${ }^{1}$ Karlsruhe Institute of Technology (KIT) \\ Hermann-von-Helmholtz-Platz 1,76344 Eggenstein-Leopoldshafen, Germany
}

simone.gianfelici@kit.edu, sara.martin@kit.edu, werner.pfrang@kit.edu, schulenberg@kit.edu, andrei.rineiski@kit.edu

\begin{abstract}
Accidents prevention and mitigation of the related consequences are mandatory for the safety assessment of the Generation IV Sodium-cooled Fast Reactors (SFRs). In order to reach these goals, the accurate evaluation of the most challenging transients, which may lead to severe accidents, is fundamental. In particular, an accurate evaluation of the degradation of the thermo-mechanical properties of the fuel pins due to irradiation, affecting the course of accidental transient conditions, is necessary. In our studies, the reference calculation route for the analysis of a severe accident in SFRs is based on the sequential use of the SAS-SFR and the SIMMER-III/IV mechanistic codes for the initiation (up to hexcan failure of a fuel sub-assembly) and the late (with core melting) phases of the scenario, respectively. Nevertheless, the module devoted to the SAS-SFR/SIMMER data transfer shows some limitations when innovative pins and core designs are considered. Having this in mind, the present paper proposes an innovative approach based on the coupling of a fuel performance code FEMAXI and the SIMMER code. When applied to severe accident studies of new reactors designs, such an approach is expected to improve the consistency of the SIMMER results as well as to extend its range of application. The FEMAXI/SIMMER coupling is described in the paper. Further, the results of the code validation against the CABRI-2 E9 experimental test, performed at the CABRI reactor using an OPHELIE-6 annular fuel pin irradiated in the PHENIX reactor, are shown and discussed. In the E9 test, a slow power ramp non-failure transient, corresponding to control rod withdrawal conditions, was investigated. New FEMAXI/SIMMER results are compared also to stand-alone SAS-SFR and SIMMER evaluations.
\end{abstract}

KEYWORDS: FEMAXI code, SIMMER code, CABRI tests, SAS-SFR code, Nuclear Reactor Safety

\section{INTRODUCTION}

The Sodium-cooled Fast Reactor (SFR) is the reactor concept selected by the Generation IV International Forum (GIF) with the largest operational experience aimed to fulfil the requirements of sustainability, reliability, safety and proliferation resistance required from the next generation of nuclear energy systems. For these reactors, accidents prevention and mitigation of the consequences are key safety aspects. An accurate evaluation of the most challenging transients, which have the potential to progress to severe accidents, is a primary concern. Since innovative fuels, like the Minor Actinide Bearing Fuels [1], and 
core designs, like the Low Void core proposed in the ESFR-SMART project [2], are expected to be employed in the next generation of SFRs, the range of applicability of the codes developed in the past for severe accident analyses needs to be reassessed and enhanced.

One relevant point is the accurate evaluation of the thermo-mechanical properties of the fuel pins due to both normal operation irradiation and accidental transient conditions. The poorer performance of the pin may play a crucial role in the evolution of the accident during transient conditions. In our studies, the current reference calculation route for the whole analysis of a severe accident in SFRs is based on the sequential use of the SAS-SFR [3-4] and the SIMMER-III/IV [5-6] mechanistic codes. The initiation phase, i.e. from the onset of the transient up to hexcan failure of a fuel sub-assembly, is evaluated by the SAS-SFR code. At this point, the calculation stops and the core state achieved can then be transferred to the SIMMER code by a devoted module, in order to evaluate the later stages of the transient up to core melting and fuel relocation.

With the studies on the innovative fuels and cores proposed by the international community, this module starts to show some limitations. When applied to a full core calculation, the transfer module can take into account only traditional designs, excluding the possibility to model internal axial fertile blankets and core zones with different heights of the fissile fuel, allowing the presence of an extended sodium plenum. In addition, the quantities related to the fuel pellets are evaluated and transferred considering only two radial nodes, a bulk inner zone and an outer thin surface zone, losing a more detailed radial description of the pellet. In case this SAS-SFR-to-SIMMER transfer module cannot be used due to the limitations mentioned above, the pin representation used in SIMMER calculation is initialized with hot design dimensions, porosity and fixed free volume gas composition, being the rest of fuel pin characteristics the as-fabricated ones.

Having this in mind, an innovative approach was developed at KIT, based on coupling the fuel performance code FEMAXI [7-8] and the SIMMER-III code. The commonly used term "coupling" in this context describes a one-directional data transfer, which technically is a chaining of the FEMAXI and the SIMMER codes. The SIMMER Over FEMAXI tool (SIMOFE) allows the user to select between several FEMAXI calculations the pin data to be transferred in a cell-wise manner to a SIMMER-III model, taking into account different fuel pin axial compositions and transferring the data for fuel multi-nodes models. During the development of this tool, a modular structure was adopted, for future extension of the tool to other fuel performance codes.

Activities are undergoing today in order to develop and validate the tool. Among them, the simulation of the CABRI-2 E9 experimental test, performed at the CABRI reactor using an OPHELIE-6 annular fuel pin irradiated in the PHENIX reactor, which will be described in this paper. In the E9 test, a slow power ramp transient, corresponding to the withdrawal of a control rod in a liquid-metal-cooled fast breeder reactor, was investigated [9].

A FEMAXI model was developed in order to evaluate the status of the pin after the irradiation in the PHENIX reactor. For the simulation of the transient in the CABRI reactor, a SIMMER-III model of the test section was set up. In order to check the verification and accuracy of the coupling, two SIMMER E9 transient calculations were performed, one with the initialization of the pin with hot fresh design data and another one using the data from the FEMAXI irradiation calculation. The results of the simulations were then compared against the experimental data and the SAS-SFR calculation performed in the framework of the JASMIN project [10]. 


\section{The SIMOFE tool}

The SIMOFE tool was developed taking advantage of the possibility to input cell-wise quantities in a SIMMER model. The data extracted directly from the FEMAXI output file are adapted and allocated for the SIMMER-III input. Considering the differences between the lagrangian FEMAXI mesh and the eulerian SIMMER mesh, which remain fixed during the calculation, in case a full core simulation is required, the tool evaluates an average axial mesh size for the core, with the aim of preserving the total fuel mass after the data transfer.

The main features of the tool can be summarized as follows:

- Supports different pin and core region designs, suggesting a mass-averaged axial mesh for the core;

- Supports multi-node fuel models, transferring radial profiles of the pellet characteristics;

- Evaluation of the thermal conductivity of the gas mixture in the free volume of the pin at the coupling time;

- Evaluation of the fuel thermal conductivity at the coupling time;

- Modular structure, for future extension to other fuel performance codes.

At the current status of the development, the axial mesh number in FEMAXI should be equal or a multiple of the SIMMER mesh, which constitutes a limitation for the user.

\section{SIMOFE assessment against CABRI-2 E9 test}

\subsection{OPHELIE-6 irradiation in PHENIX reactor}

The E9 test [11-12] was performed with an industrial design pin with annular pellets called OPHELIE-6. The main data for this design are reported in Table I. The pin was previously irradiated in the PHENIX reactor up to a maximum burnup of $\sim 5$ at. $\%$.

Table I. OPHELIE-6 design data

\begin{tabular}{|l|c|c|}
\hline & OPHELIE-6 & Unit \\
\hline Fuel design & Hollow pellet & - \\
\hline Pellet inner diameter & 2 & $\mathrm{~mm}$ \\
\hline Pellet outer diameter & 7.27 & $\mathrm{~mm}$ \\
\hline Clad inner diameter & 7.5 & $\mathrm{~mm}$ \\
\hline Clad outer diameter & 8.65 & $\mathrm{~mm}$ \\
\hline Fissile length & 750 & $\mathrm{~mm}$ \\
\hline Fuel density & 95.5 & $\%$ \\
\hline $\mathrm{Pu} /(\mathrm{U}+\mathrm{Pu})$ ratio & 14.503 & - \\
\hline Clad material & SS 316 cold work & - \\
\hline
\end{tabular}

In order to simulate the irradiation of the OPHELIE-6 pin in the PHENIX reactor, a FEMAXI model was set up, simulating the irradiation conditions. The fuel column was simulated by 17 axial meshes. The results were then compared to the available Post Irradiation Examination (PIE) data at the End of Irradiation (EOI), as reported in Table II. FEMAXI results are in reasonable agreement with the PIE data. For this reason, the data have been processed with the SIMOFE tool for the SIMMER-III code. 
Table II. EOI state for the OPHELIE-6 pins: Experimental vs. FEMAXI vs. SAS-SFR

\begin{tabular}{|l|c|c|c|c|}
\hline & Exp. data & FEMAXI & SAS-SFR & Unit \\
\hline Fission gas generated & - & 1.4814 & 1.4604 & $\mathrm{~g}$ \\
\hline Fission gas release & 53.1 & 52.6 & 53.2 & $\%$ \\
\hline Plenum pressure at $400^{\circ} \mathrm{C}$ & 1.2 & 1.1 & 1.01 & $\mathrm{MPa}$ \\
\hline PPN hole over external radius & 33 & 31 & 31 & $\%$ \\
\hline PPN burnup & 4.89 & 4.9 & 4.74 & at. $\%$ \\
\hline
\end{tabular}

\subsection{SIMOFE verification}

Following the FEMAXI nodalization, a SIMMER-III model of the CABRI test section was set up with 17 axial meshes for the fuel column, 10 radial nodes for the fuel pellet and one radial node for the cladding. Based on the experimental observations, radial heat losses were considered in the SIMMER calculations. As mentioned above, two inputs were prepared, one for the stand-alone SIMMER calculation, modelling a fresh fuel pin with hot dimensions (so-called SIMMER case) and another one importing the EOI data from the FEMAXI calculation (so-called FEMAXI/SIMMER case). A verification of the correct data transfer between both codes was performed by checking relevant quantities, as reported in Table III. The total mass of the fissile column is a critical value, since the SIMMER user cannot specify it explicitly but through the cell dimensions, fuel volume fractions, temperature and the porosity. The slight discrepancy between the two codes $(0.1 \mathrm{~g})$, due to mainly an approximation of the plutonium and uranium densities at the beginning of the calculation in the FEMAXI input, gives a reasonable confidence on the correct implementation of the tool. In addition, the retained fission gas (FG) over fuel mass ratio was checked. A discrepancy was detected, probably due to approximation errors when performing the conversion from retained $\mathrm{FG}$ atoms $/ \mathrm{cm}^{3}$, the FEMAXI output, to $\mathrm{FG} /$ fuel mass ratio, the SIMMER input variable.

Table III. SIMOFE verification: FEMAXI-to-SIMMER data transfer

\begin{tabular}{|l|c|c|c|c|}
\hline & FEMAXI & SIMMER & FEMAXI/SIMMER & Unit \\
\hline Total fissile mass & 301.9 & 301.8 & 301.8 & $\mathrm{~g}$ \\
\hline Retained FG/fuel mass ratio & $0(\mathrm{BOI})$ & 0 & - & - \\
\hline & $0.0025(\mathrm{EOI})$ & - & 0.0026 & - \\
\hline Hot fissile length & $0.765(\mathrm{BOI})$ & 0.765 & - & $\mathrm{m}$ \\
\hline & $0.772(\mathrm{EOI})$ & - & 0.772 & $\mathrm{~m}$ \\
\hline
\end{tabular}

\subsection{E9 test conditions}

In the E9 test, performed in the CABRI research reactor in 1991, the pin was submitted to a power ramp of around $1.1 \%$ nominal power rise per second $(\mathrm{Pn} / \mathrm{s})$, similar to a control rod withdrawal accident. At Peak Power Node (PPN) the linear power started from a nominal condition of $603 \mathrm{~W} / \mathrm{cm}$ reaching after $118 \mathrm{~s}$ a final value at the End Of the Transient (EOT) of $1347 \mathrm{~W} / \mathrm{cm}$. The fuel pin did not fail during the test, but a large fuel melt cavity formation was observed in Post Test Examinations (PTE).

The initial experimental test conditions are summarized in Table IV, together with the initial parameters of the SIMMER, FEMAXI/SIMMER and SAS-SFR codes. Both SIMMER calculations are with the same input for the power and flow rate conditions; however, the different initial fissile lengths lead to different 
Linear Heat Rates (LHR) at PPN. Because of neutron spectrum variations in the test section of the CABRI facility, a radial depression of the power profile has been taken into account.

Table IV . E9 Initial tet conditions

\begin{tabular}{|l|c|c|c|c|c|}
\hline & Experiment & SIMMER & FEMAXI/SIMMER & SAS-SFR & Unit \\
\hline Total power & $36297 \pm 1316$ & 35680 & 35680 & 38980 & $\mathrm{~W}$ \\
\hline Power in fuel pellets & $35680 \pm 1106$ & 35680 & 35680 & 38200 & $\mathrm{~W}$ \\
\hline Power in fissile & - & 100 & 100 & 94.7 & $\%$ \\
\hline Power in fertile & - & 0.0 & 0.0 & 5.3 & $\%$ \\
\hline Gamma heating & $2.1 \pm 0.2$ & 0.0 & 0.0 & 2.0 & $\%$ \\
\hline BFC coolant temperature & $663.0 \pm 5.1$ & 668.1 & 668.1 & 662.6 & $\mathrm{~K}$ \\
\hline $\begin{array}{l}\text { Na } \Delta \text { T across fuel } \\
\text { column }\end{array}$ & $186.6 \pm 3.0$ & 191.7 & 191.3 & 189.2 & $\mathrm{~K}$ \\
\hline Flow rate & $0.642 \pm 0.025$ & 0.651 & 0.651 & 0.651 & $\mathrm{~m} / \mathrm{h}$ \\
\hline LHR at PPN & $603 \pm 25$ & 607 & 603 & 621.4 & $\mathrm{~W} / \mathrm{cm}$ \\
\hline Fissile column length & $775 \pm 2$ & 765 & 772 & 766 & $\mathrm{~mm}$ \\
\hline Radial power depression & 0.45 & 0.4 & 0.4 & 0.4 & - \\
\hline
\end{tabular}

\subsection{Comparison of the calculations results with the experiment}

The calculated sodium temperature during the transient at the Top of the Fissile Column (TFC), shown in Figure 1, is in good agreement with the data of the six thermocouples present in the same location of the test channel, assuring about the correct modelling of the radial heat losses. In agreement with the outcome of the experiment, no pin failure was predicted by any code. As reported in Table V, even though the location at which the fuel melting starts is very similar, the dynamic of the fuel melting onset is different for different codes. SIMMER with fresh fuel data calculates fuel melting at $10 \mathrm{~s}$ after Beginning of Transient (BOT), $6 \mathrm{~s}$ earlier than FEMAXI/SIMMER. This can be explained with Figure 2, where the average fuel temperature for the SIMMER calculation is higher $(\sim 30 \mathrm{~K})$ than the FEMAXI/SIMMER case. Since the fuel pin status in this case is that of a fresh fuel, the central hole is smaller and the gap is still open unlike the irradiated case, leading to higher fuel temperatures. In the SAS-SFR calculation, the fuel temperature is lower, and a delayed, in respect to SIMMER; fuel melting onset is predicted at $25 \mathrm{~s}$ into the transient. After the fuel melting onset, the temperature trends between SAS-SFR and SIMMER differ significantly. The increase in the average fuel temperatures calculated by SAS-SFR at about $25 \mathrm{~s}$ might be explained by fuel restructuration as indicated by the sudden decrease in the inner fuel radius and in the gap conductance.

Table V. E9 Fuel melting onset

\begin{tabular}{|l|c|c|c|c|}
\hline & SIMMER & FEMAXI/SIMMER & SAS-SFR & Unit \\
\hline Fuel melting onset time from BOT & 10.17 & 16.19 & 25 & $\mathrm{~s}$ \\
\hline Fuel melting onset location & 34.44 & 34.76 & 33.95 & $\mathrm{~cm}$ \\
\hline Fuel pin failure & no failure & no failure & no failure & - \\
\hline
\end{tabular}




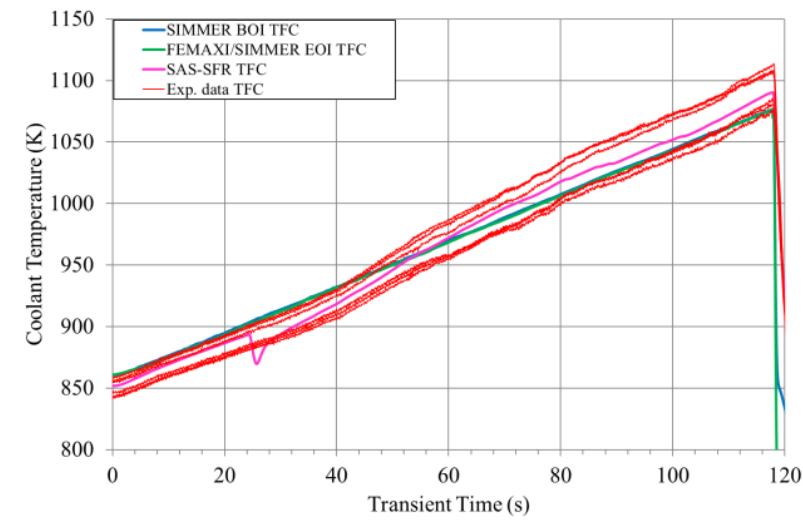

Figure 1. Na temperature at TFC



Figure 2. Average fuel temperature at PPN

The different average fuel temperature trends are reflected in the fission gas release, shown in Figure 3 (no data from the SIMMER case are reported, since the input of the FG/fuel mass ratio for this case was zero). Initially similar up to the melting onset, the FEMAXI/SIMMER calculation underestimates the fission gas release during the transient, giving indications for the improvement of the cavity model. Another limitation is presented after the transient, ended at $118 \mathrm{~s}$. If the pin is intact, the volume of the cavity does not allow mass transfer outside the pellet. For this reason, when the fuel starts to solidify after the transient, the released FP gasses in the cavity are incorporated back in the fuel matrix, leading to a decrease of the fission gas release.



Figure 3. Fission gas release

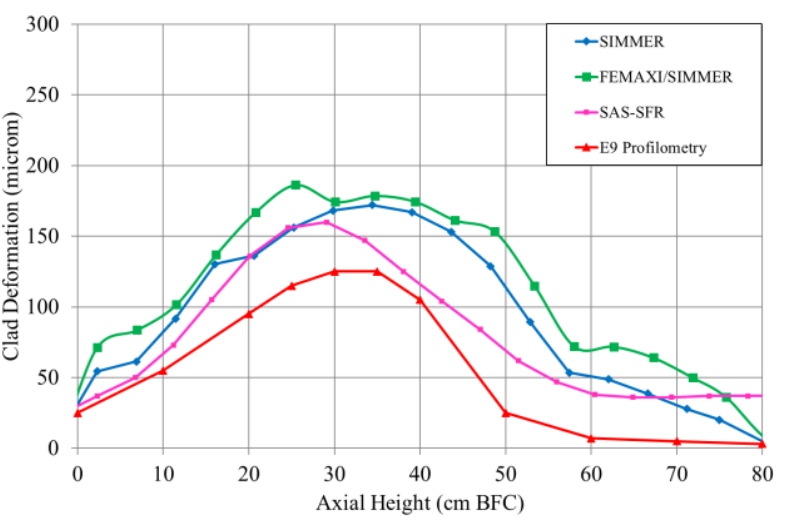

Figure 4. Cold cladding radial deformation

The axial profile of the cladding radial deformation at cold conditions is reported in Figure 4. Due to the nature of the SIMMER-III code, which performs the simulation with a fixed eulerian mesh, axial deformations are not allowed. Because of the mass conservation, this behavior leads to a systematic overestimation of the radial displacements of the solid materials. For this reason, the two SIMMER calculations share similar trends with the experimental data, but at higher values. 


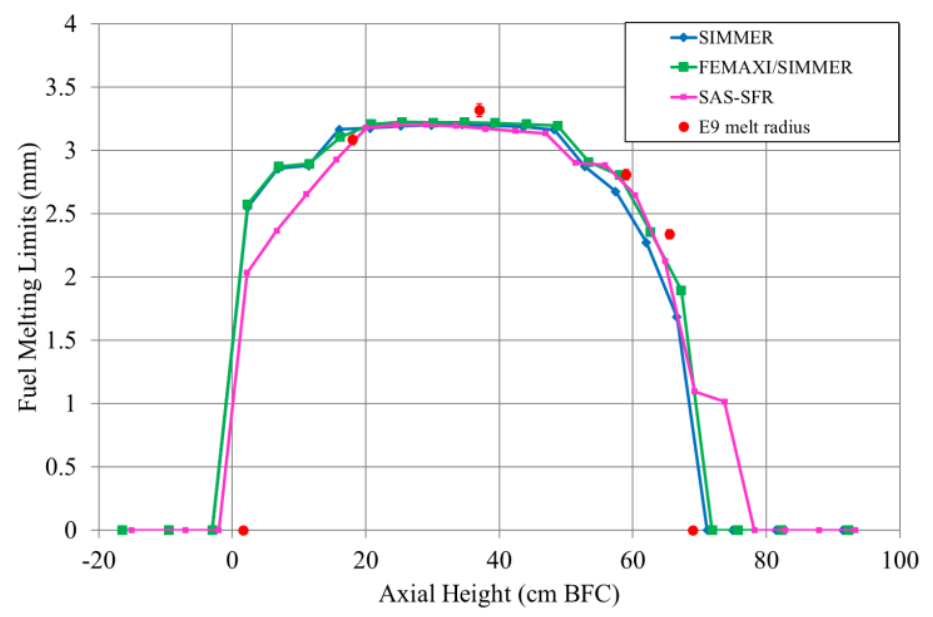

Figure 5. Fuel melting cavity radius at EOT

In Fig. 5 the final cavity radius at the end of the test is shown. Both FEMAXI/SIMMER and SAS-SFR predict reasonably well the final limits of the cavity detected during the E9 experiment. Even if the fuel melting process starts earlier for the SIMMER case calculation, the better thermal performances of the fresh fuel-pin configuration lead to an underestimation of the final cavity volume in the upper part of the fuel column.

\section{CONCLUSIONS}

In the view of the next generation of nuclear reactor technology, we are trying to improve and extend the capabilities of the codes developed in the past. To cope with the new fuel designs, fuel materials, core designs and increased safety levels demanded, tools and codes are being extended and developed to assess the main safety aspects of the Generation IV reactors. In order to have a reliable deployment of these new tools, a validation process against the available experimental data is indeed needed.

With this in mind, a new tool for the coupling between the FEMAXI and the SIMMER code was developed, which allows to initialize the fuel pin characteristics with data evaluated after the irradiation in a SFR so that it is possible to take into account the degraded performances when simulating transient accident conditions. The tool overcomes also some limitations of the SAS-SFR-to-SIMMER transfer tool, which was developed with a focus on the traditional SFR core design. As a start for the validation process, the SIMOFE tool was deployed to simulate the E9 slow power ramp test performed in the CABRI reactor for the CABRI-2 experimental campaign.

The calculation required the setting up of a simulation with the FEMAXI code of the irradiation in the PHENIX reactor of the OPHELIE- 6 set of pins, target of the transient test. The results are in reasonable agreement with the available PIE data. The output of this calculation constitutes the basis for the data transfer to SIMMER with the SIMOFE tool. The correct implementation of the data transfer was verified.

The effect of the irradiated fuel data on the SIMMER E9 evaluation was evaluated performing two different SIMMER-III calculations, one with the classical approach and one taking advantage of the SIMOFE tool. The results of these calculations were compared to the available experimental data and the reference code SAS-SFR calculation.

The SIMMER-III and the SAS-SFR codes showed differences in the dynamic of the fuel melting onset and of the fuel average temperature at PPN during the transient due to the fuel restructuring as predicted by SAS-SFR calculation. This is reflected in the fission gas release trend, which is underestimated by the 
SIMMER-III code. Improvements are needed for the cavity model of the SIMMER-III code, in particular in the assessment of the fission gas mass transfer from the fuel to the free volume during slow transients. Another relevant difference is in the different mesh systems, since the SIMMER code does not allow axial variations of the structures. For this reason, the final cladding radial deformation is overestimated. Regarding the final fuel melting cavity, the FEMAXI/SIMMER and the SAS-SFR calculations are closer to experimental data with respect to the SIMMER case calculation.

This calculation constitutes the first validation test for the SIMOFE tool. Further validation tests are needed, especially when pin failure and fuel relocation inside the channel are involved. Regarding the cavity formation, the results obtained with the SIMOFE tool showed an improvement from the standalone SIMMER calculation, being closer to the experimental data in the assessment of the final fuel melting extension. These improvements provide a solid bases for future FEMAXI/SIMMER application to safety studies of innovative SFRs designs.

\section{REFERENCES}

1. A. Gallais-During, F. Delage, S. Béjaoui, S. Lemehov, J. Somers, D. Freis, W. Maschek, S. Van Til, E. D'Agata, C. Sabathier, "Outcomes of the PELGRIMM project on Am-bearing fuel in pelletized and spherepac forms", Journal of Nuclear Materials, 512, pp. 214-226 (2018).

2. A. Rineiski, C. Meriot, M. Marchetti, J. Krepel, "Core Safety Measures in ESFR-SMART", Proceedings of the PHYSOR 2018, Cancun, Mexico, April 22-26, 2018 (2018).

3. U. Imke, D. Struwe, H. Niwa, I. Sato, F. Camous, D. Moxon, "Status of the SAS4A-code development for consequence analysis of core disruptive accidents", Proceedings of an International Topical Meeting. Sodium Cooled Fast Reactor Safety, Obninsk, Russia, October 1994, pp. 232-241 (1994).

4. R. Kruessmann, A. Ponomarev, W. Pfrang, D. Struwe, J. Champigny, B. Carluec, D. Schmitt, D. Verwaerde, "Assessment of SFR reactor safety issues: Part II: Analysis results of ULOF transients imposed on a variety of different innovative core designs with SAS-SFR", Nuclear Engineering and Design, 285, pp. 263-283 (2015).

5. S. Kondo, Y. Tobita, K. Morira, N. Shirakawa, "SIMMER-III: an advanced computer program for LMFBR severe accident analysis" Proceedings of the International Conference on Design and Safety of Advanced Nuclear Power Plant (ANP'92), Tokyo, Japan, October 25-29, Vol. IV, pp. 40.5.140.5.11 (1992).

6. H. Yamano, S. Fujita, Y. Tobita, I. Sato, H. Niwa, "Development of a three-dimensional CDA analysis code: SIMMER-IV and its first application to reactor case", Nuclear Engineering and Design, 238(1), pp. 66-73 (2008).

7. M. Suzuki, H. Saitou, Y. Udagawa, Light Water Reactor Fuel Analysis Code FEMAXI-7; Model and Structure, JAEA-Data/Code 2010-035, Japan Atomic Energy Agency (JAEA), Japan (2010).

8. T. Okawa, I. Tatewaki, T. Ishizu, H. Endo, Y. Tsuboi, H. Saitou, "Fuel behavior analysis code FEMAXI-FBR development and validation for core disruptive accident", Progress in Nuclear Energy, 82, pp 80-85 (2015).

9. J. Charpenel, L. Francette, I. Sato, D. Struwe, W. Pfrang, "Fuel Pin Behavior under the Slow Power Ramp Transients in the CABRI-2 Experiments", Nuclear Technology, 130, pp. 252-271 (2000).

10. S. Perez-Martin, W. Pfrang, N. Girault, L. Cloarec, L. Laborde, M. Buck, V. Matuzas, A. Flores y Flores, P. Raison, A.L. Smith, N. Mozzani, F. Feria, L. Herranz, B. Farges, "Development and assessment of ASTEC-Na fuel pin thermo-mechanical models performed in the European JASMIN project", Annals of Nuclear Energy, 119, pp. 454-473 (2018).

11. M. Haessler, D. Struwe, A.T.D. Butland, N. Nonaka, I. Sato, J. Papin, "The CABRI 2 programme overview and results", Proceedings of the International Fast Reactor Safety Meeting, Snowbird, USA, August 12-16. Vol. II, pp. 209-221 (1990).

12. J. Papin, Comprehensive Nuclear Materials, Ch. 2.24 pp. 609-634, Elsevier (2012). 